\title{
RESEARCH HIGHLIGHT IL-18: throwing off the shackles to boost anti-tumor immunity
}

\author{
Karen O. Dixon (iD) and Vijay K. Kuchroo ${ }^{1}$ \\ Cell Research (2020) 30:831-832; https://doi.org/10.1038/s41422-020-00396-3
}

\begin{abstract}
In a new study in Nature, Zhou et al. identify IL-18BP as a novel soluble immune checkpoint, and describe engineering of a decoy-resistant IL-18 to overcome this barrier and drive potent anti-tumor immunity.
\end{abstract}

Cytokines are soluble molecules important in cell signaling and communication, with the ability to orchestrate the immune response in autoimmunity, infection and cancer. The discovery of the potent tumoricidal activity of interferons paved the way for intense clinical investigation of several inflammatory cytokines in preclinical models, including IFN- $\mathrm{a}^{1}$ and $\mathrm{IL}-2{ }^{2}$ with cytokine therapy historically being an important treatment modality for cancer. While the use of these cytokines was a significant milestone in cancer immunotherapy, as a monotherapy they have not fulfilled the promise of efficacy seen in preclinical models. In addition to a wide range of toxicities, the low response rate has largely relegated the use of these therapeutics in favor of immune checkpoint inhibitors, including anti-CTLA-4 and anti-PD-1, which have shown profound clinical benefits in a number of patients that were otherwise refractory to conventional anti-tumor therapies. However, only $\sim 13 \%$ of patients respond to these therapies, ${ }^{3}$ and despite a major effort to develop "second line" immunotherapies, there has yet to be significant additive clinical benefit beyond existing strategies. In this context, there is a renewed interest in potentiating the anti-tumor effect of inflammatory cytokines in combination with existing immunomodulatory agents, including anti-PD-1. ${ }^{4}$ To circumvent the earlier caveats with cytokine immunotherapy, such as pleiotrophism and low response rate, cytokines are now also being engineered to generate "superkines" sustaining their anti-cancer efficacy or tailoring their receptor-binding affinity. This is best exemplified by engineered IL-2-like molecules with augmented affinity for IL-2R $\beta$ and increased effector $T$ cell function. ${ }^{5}$

In a new study published in Nature, the authors sought to explore novel cytokine circuits within the tumor microenvironment (TME) leveraging existing single-cell tumor-infiltrating lymphocyte (TIL) datasets. ${ }^{6}$ Using this approach, they found that transcripts of IL18R1 (encoding IL-18Ra) and IL18RAP (encoding IL$18 \mathrm{R} \beta$ ) were enriched in activated and dysfunctional CD8 ${ }^{+}$TILs. Despite the promising expression profile of the receptor, IL-18 had previously been explored in a phase II clinical trial which was prematurely terminated due to low clinical efficacy, failing to recapitulate the strong anti-tumor immunity seen in preclinical models with recombinant IL-18.

IL-18 is a member of the IL- 1 family of cytokines, and analogous to IL-1 $\beta$ is a potent inflammatory cytokine. As such, both are tightly regulated and synthesized as precursors, which, in permissive conditions, are cleaved and released as mature IL-1 $\beta$ and IL-18. Upon secretion these cytokines are further regulated by decoy receptors IL-1Ra and IL-18BP, respectively. The affinity of
IL-18BP for IL-18 is $\sim 10,000$ times greater than that of IL-18Ra, leading the authors to hypothesize that IL-18BP might be inhibiting the activity of IL-18 in the TME. The authors found that levels of IL-18BP were elevated in both human and murine tumors in an IFN- $\gamma$-dependent manner. Interestingly, IL-27, a key cytokine driving expression of the co-inhibitory module on CD8 $\mathrm{TILs}^{7}$ is also a potent inducer of IL-18BP. Therefore, in addition to directly regulating the induction of surface inhibitory receptors on $\mathrm{CD}^{+}$ $\mathrm{T}$ cells, IL-27 contributes to $\mathrm{CD} 8^{+} \mathrm{T}$ cell dysfunction in the TME through its action on myeloid cells, promoting a negative feedback loop through induction of a number of cell surface and soluble inhibitors, including IL-18BP.

To overcome the inhibitory effect of IL-18BP, the authors performed a yeast display screening of IL-18 variants to identity mutants which could bind the IL-18Ra, but not IL-18BP. This "decoy-resistant" IL-18 (DR-18) showed remarkable results as a monotherapy and synergistic effect with anti-PD-1, expanding memory precursor $\mathrm{CD}^{+} \mathrm{T}$ cells and mitigating $\mathrm{T}$ cell exhaustion with lower levels of TOX (Fig. 1). TOX is a key transcription factor critical for the transcriptional program of $\mathrm{CD}^{+} \mathrm{T}$ cell exhaustion downstream of nuclear factor of activated T cell (NFAT) signaling. ${ }^{8}$ Interestingly, NFAT proteins have a dichotomous role regulating $T$ cell activation while also participating in the transcriptional program of $\mathrm{CD}^{+} \mathrm{T}$ cell exhaustion. The findings from this study suggest that NFAT activation concomitant with NF-KB signaling through IL-18R is sufficient to deviate from an exhaustion trajectory, sustaining effector $\mathrm{T}$ cell responses for anti-tumor immunity. While further studies are warranted to fully investigate this, it would provide compelling rationale to utilize the mutant IL18 (DR-18) in combination with anti-PD-1, directing the expanded precursor population seen in PD-1 blockade, towards a potent effector lineage fate.

Importantly, through its ability to license natural killer (NK) cells DR-18 could also act on MHC class I-deficient tumors which are resistant to conventional immune checkpoint therapy (Fig. 1). Currently, a number of NK checkpoint molecules, including KIR and CD94, are being tested in clinical trials. It will be interesting to test the potential synergy of these therapies together with DR-18, which could have significant efficacy in "cold tumors" where there is a current unmet clinical need.

While it appears that DR-18 is well tolerated and efficacious at low doses, immune-related adverse events will need to be thoroughly addressed before clinical translation. Dysregulation of $\mathrm{IL}-18$ is associated with autoimmune diseases including inflammatory bowel disease, granulomatosis, and SLE, where levels of free IL-18 (IL-18 not bound by IL-18BP) correlate with disease progression. Notably, loss-of-function mutations in IL18BP can lead to excessive NK cell activation resulting in severe hepatitis. $^{9}$ Furthermore, similar to IL-1 $\beta, \mathrm{IL}-18$ induces IL-17

\footnotetext{
${ }^{1}$ Ann Romney Center for Neurologic Diseases and Evergrande Center for Immunologic Diseases, Brigham and Women's Hospital, Harvard Medical School, Boston, MA, USA Correspondence: Vijay K. Kuchroo (vkuchroo@evergrande.hms.harvard.edu)
}

Published online: 18 August 2020 


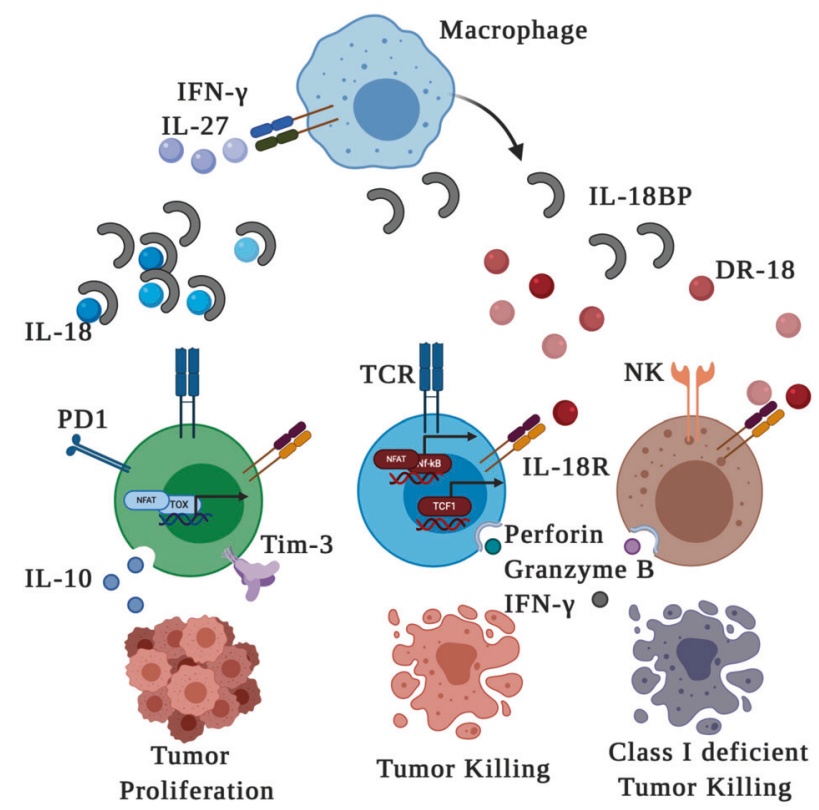

Fig. 1 DR-18 remodels the TME to promote effective tumor cell clearance. In the TME, native IL-18 induces IFN- $\gamma$ and IL-27 which act on macrophages to induce the decoy receptor IL-18BP in a negative feedback loop, neutralizing active IL-18. Engineered DR-18 evades IL-18BP but can still exert its function through IL-18R expressed on activated T cells and NK cells. NFAT activation, concomitant with NF$\kappa B$ activation downstream of IL-18R signaling, curtails TOX expression, sustaining TCF1 expression and promoting effector T cell generation. DR-18 mitigates NK cell dysfunction in MHC class I-deficient tumors, leading to efficient cytolysis of malignant cells. production in $\mathrm{T}$ cells, promoting autoimmune responses to specific antigens. Interestingly, in humans, unusually elevated levels of IL-18 have been observed in haemophagocytic syndromes. ${ }^{10}$ Macrophage activation syndrome has been observed in patients treated with conventional checkpoint immunotherapy with anti-PD-1, and one could speculate enhanced risk with DR-18. Clinical application of DR-18 either as monotherapy or in combination with other biological agents will require further studies to bring it to fruition as a major contributor for the treatment of malignancy. Whereas existing checkpoint-based therapy blocks inhibitory receptors expressed on T cells, and their efficacy has reached a ceiling beyond which increased efficacy may not be achievable, soluble engineered molecules like DR-18 will provide additional tools to complement current checkpointbased immunotherapies. The challenge, however, will be to see how to identify the clinical indications and patient populations where the combination will have meaningful impact.

\section{ADDITIONAL INFORMATION}

Competing interests: The authors declare no competing interests.

\section{REFERENCES}

1. Kirkwood, J. M. et al. J. Clin. Oncol. 14, 7-17 (1996).

2. Fyfe, G. et al. J. Clin. Oncol. 13, 688-696 (1995).

3. Haslam, A. \& Prasad, V. JAMA Netw. Open 2, e192535 (2019).

4. Berraondo, P. et al. Br. J. Cancer 120, 6-15 (2019).

5. Levin, A. M. et al. Nature 484, 529-533 (2012).

6. Zhou, T. et al. Nature 583, 609-614 (2020).

7. Chihara, N. et al. Nature 558, 454-459 (2018).

8. Scott, A. C. et al. Nature 571, 270-274 (2019).

9. Belkaya, S. et al. J. Exp. Med. 216, 1777-1790 (2019).

10. Weiss, E. S. et al. Blood 131, 1442-1455 (2018). 